\title{
Research Article \\ Effect of Microwave Irradiation on Polyvinyl Alcohol as a Carrier of Silver Nanoparticles in Short Exposure Time
}

\author{
Dat Tan Nguyen (D), Khanh Loan Ly, Nam Minh-Phuong Tran, Minh Hieu Ho, \\ Trang Thi-Phuong Tran, Thi-Hiep Nguyen $\mathbb{1}$, Dang Ngọc Thao Nhi, and Van Toi Vo \\ Department of Biomedical Engineering, Vietnam National University-Ho Chi Minh City (VNU-HCM)-International University, \\ Ho Chi Minh City 700000, Vietnam \\ Correspondence should be addressed to Thi-Hiep Nguyen; nthiep1981@gmail.com
}

Received 19 July 2019; Accepted 20 November 2019; Published 5 December 2019

Guest Editor: Chao Zhao

Copyright (c) 2019 Dat Tan Nguyen et al. This is an open access article distributed under the Creative Commons Attribution License, which permits unrestricted use, distribution, and reproduction in any medium, provided the original work is properly cited.

\begin{abstract}
Microwave heating of $\mathrm{Ag}^{+}$in polyvinyl alcohol (PVA) is an effective method to synthesize silver nanoparticles (AgNPs), a broadrange antibacterial agent. However, the microwave may cause PVA solution to dry out and degrade. This study is aimed at investigating the effect of microwave irradiation on different concentrations of PVA in PVA/ $\mathrm{Ag}^{+}$solution in short periods of exposure. Fourier-transform infrared (FTIR) spectroscopy was employed to evaluate chemical changes of samples with different exposure times and PVA concentration. The results confirm the redox reaction of $\mathrm{Ag}^{+}$with PVA. In addition, $\mathrm{Ag}^{+}$reduces the rate of hydrolysis of PVA, and the ether bridges are limited by the spatial structure.
\end{abstract}

\section{Introduction}

Antibiotic resistance has become one of the most significant health threats because microbes evolve rapidly against our antibiotics while the development of new antibiotics takes decades. Consequently, the old drugs-including metal and their salts that were used to treat infections before the era of antibiotics dominance-have once again become the research focus [1]. Among metals such as silver, copper, zinc, or gold, silver, whose applications have been widely studied $[2,3]$, has a broad range of antibacterial properties and has relatively low toxicity. Silver nanoparticles (AgNPs) - whose scale is from 1 to $100 \mathrm{~nm}$-also exhibit good biological interaction. However, many studies proved that inappropriate size and concentration of AgNPs are toxic to mammalian cells [4-6]. Therefore, rather than using pure AgNPs, a carrier or stabilizer to control the size and enhance the surface interaction is generally necessary [7-11].

Polyvinyl alcohol (PVA) - a hydroxy polymer with the idealized formula $\left[\mathrm{CH}_{2} \mathrm{CH}(\mathrm{OH})\right]_{n}$ - possesses many excellent features such as biocompatibility, biodegradability, water-solubility, and gel-forming. However, they are thermally unstable. During the heat treatment, two major stages occur: elimination reactions and chain scission and cyclization [12]. Elimination reaction produces reactive carbon atoms along the polymer chain, which makes PVA highly reactive to other chemicals during the thermal process. Taking the advantages of this phenomenon, adding $\mathrm{Ag}^{+}$ions in PVA solution will cause a redox reaction between $\mathrm{Ag}^{+}$, an oxidant, and reactive PVA, a reductant. In previous studies, PVA was proven to be a potential carrier/stabilizer for the synthesis of AgNPs [9]. Moreover, thermal stimulation of PVA could occur without the need of additional catalyst or cross-linker that may induce harmful effects to the body [9]

There are two primary techniques to induce reactions: conventional heating and microwave heating. Compared with conventional heating, microwave heating is a more versatile and economical method with reduced reaction times [13]. Bernal et al. investigated the effect of microwave on PVA with ethylene glycol as the solvent from $4 \mathrm{~min}$ to 60 min [12]. However, overexposure of microwave irradiation causes parching and total degradation of PVA. In the 
TABLE 1: List of samples.

\begin{tabular}{lccc}
\hline Abbreviation & PVA concentration (\%) & Exposure time (s) & AgNO $_{3}$ added \\
\hline P8 & 8 & 0 & No \\
P8T60 & 8 & 60 & No \\
P8T90 & 8 & 90 & No \\
P6T60Ag & 6 & 60 & Yes \\
P8T60Ag & 8 & 60 & Yes \\
P10T60Ag & 10 & 60 & Yes \\
P6T90Ag & 6 & 90 & Yes \\
P8T90Ag & 8 & 90 & Yes \\
P10T90Ag & 10 & 90 & Yes \\
\hline
\end{tabular}

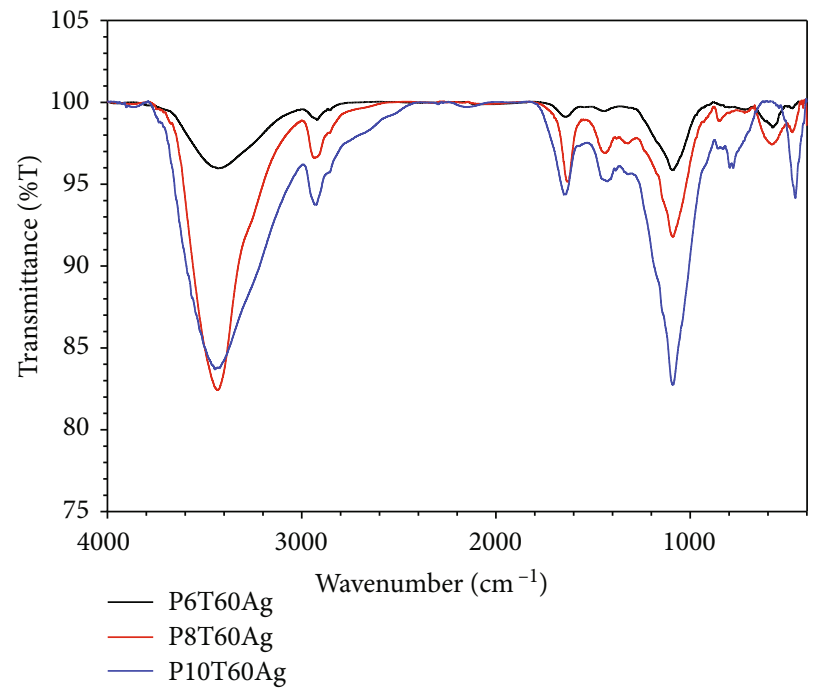

(a)

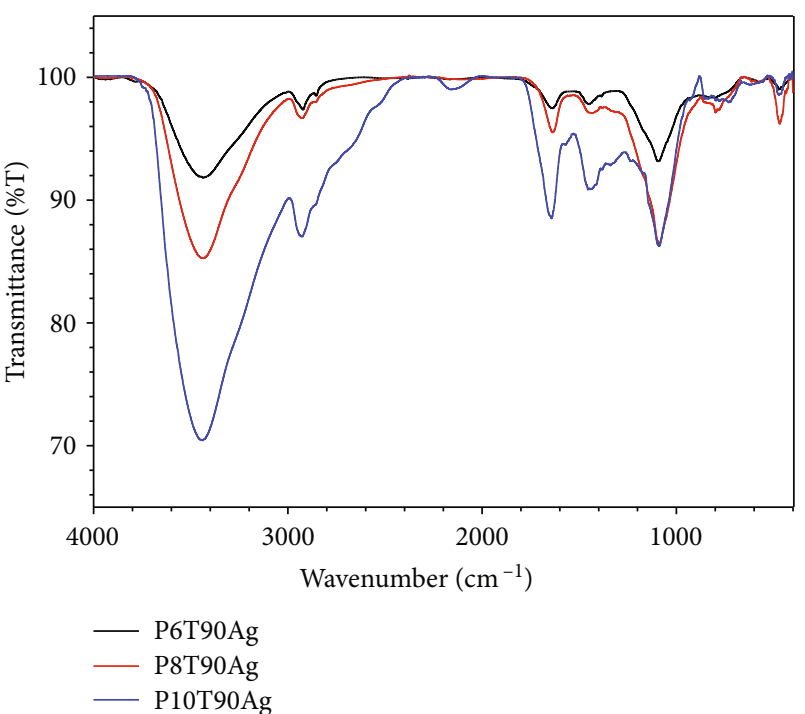

(b)

Figure 1: FTIR spectra of (a) P6T60Ag, P8T60Ag, and P10T60Ag and (b) P6T90Ag, P8T90Ag, and P10T90Ag.

application as a carrier of AgNPs, the exposure time of microwave for PVA is a significant factor, and its effects have not been well-understood yet.

In this study, we investigate the effect of microwave irradiation on different concentrations of PVA in PVA/ $\mathrm{Ag}^{+}$ solution in short periods of exposure.

\section{Materials and Methods}

2.1. Materials. Polyvinyl alcohol (PVA; hydrolysis degree of 99.0-99.8\%) was purchased from Sigma-Aldrich, USA. Silver nitrate $\left(\mathrm{AgNO}_{3} \geq 99 \%\right)$ was obtained from Guangdong, China. The microwave oven is a magnetron MM250 (Whirlpool Corp., USA).

2.2. Sample Preparation. Typically, aqueous solutions of $6 \mathrm{wt} \%, 8 \mathrm{wt} \%$, and $10 \mathrm{wt} \%$ of fully hydrolyzed PVA were prepared by dissolving PVA in distilled water at $75^{\circ} \mathrm{C}$. The $1 \%$ silver nitrate solution was added to the PVA solution so that the silver concentration was up to $120 \mathrm{ppm}$. Subsequently, the PVA solution was microwaved at $800 \mathrm{~W}$ for determined periods. All samples are listed below in Table 1.

2.3. FTIR Measurement. Samples were freeze-dried into powder in order to avoid the interference of the hydrogen bonding of water molecules. Then, their FTIR spectra were obtained using a FTIR analyzer (PerkinElmer Spectrum GX, USA).

\section{Results and Discussion}

The original PVA was characterized with a broad, strong $\mathrm{O}-\mathrm{H}$ stretching within the range $3700-3000 \mathrm{~cm}^{-1}$, a medium aliphatic C-H stretching peak within the 2920$2930 \mathrm{~cm}^{-1}$ region, and along with $\mathrm{C}=\mathrm{O}$ stretch (around $\left.1640 \mathrm{~cm}^{-1}\right), \mathrm{C}-\mathrm{O}$ stretch $\left(1088 \mathrm{~cm}^{-1}\right)$, and $\mathrm{CH}_{2}$ and $\mathrm{CH}_{3}$ bending $\left(1438 \mathrm{~cm}^{-1}\right)$. All the peaks of PVA spectrum reappeared in other samples (Figures 1 and 2), which proved that the effects occurred partially in the PVA structure and PVA can still maintain its properties under microwave in short periods of exposure. 


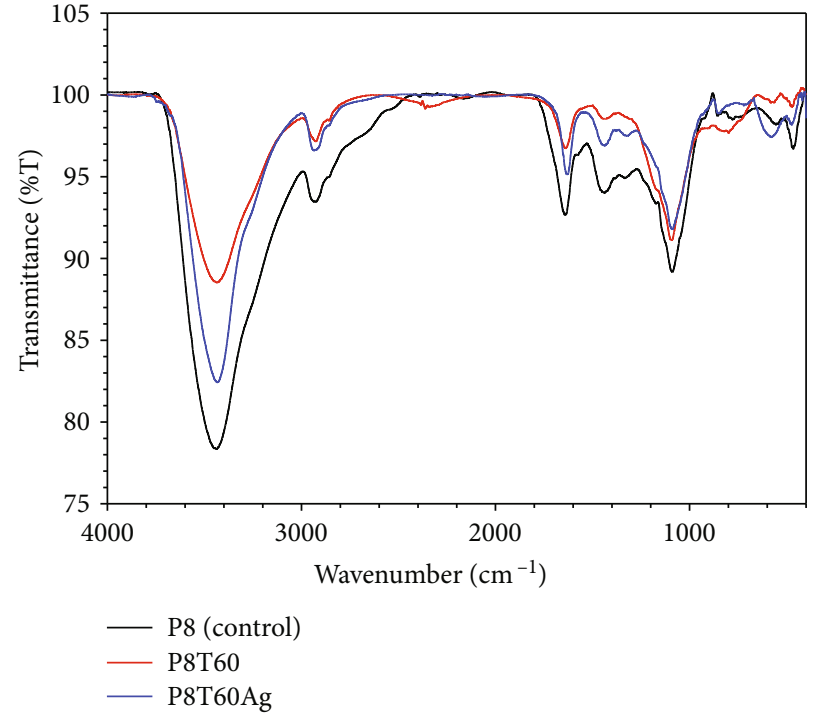

(a)

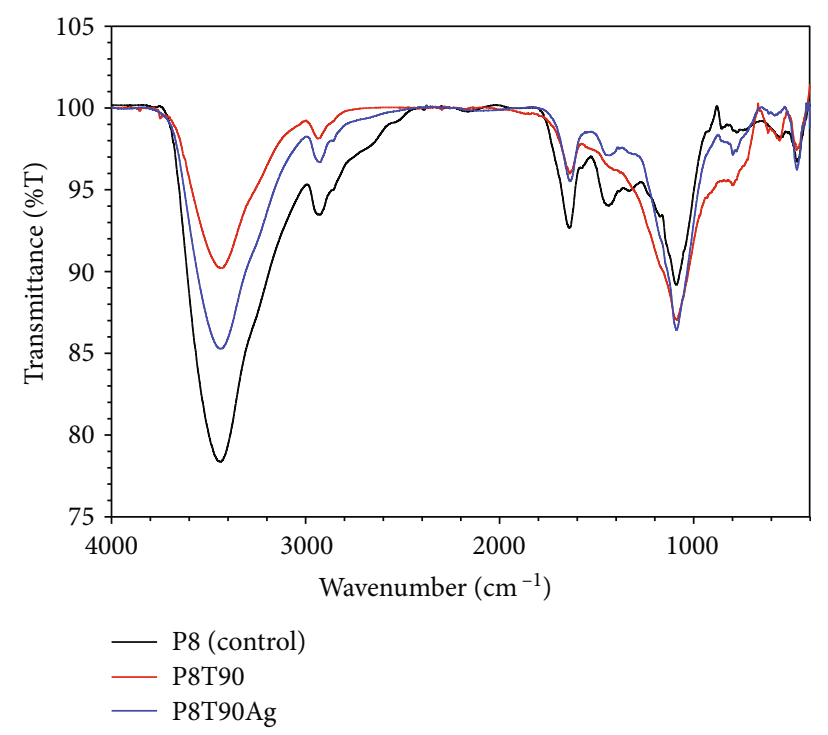

(b)

Figure 2: FTIR spectra of (a) P8, P8T60, and P8T60Ag and (b) P8, P8T90, and P8T90Ag.

Figure 3 displays the FTIR spectra of microwaved and nonmicrowaved of freeze-dried of $8 \mathrm{wt} \%$ PVA solution. As the time of microwave exposure increases, the absorbance of $\mathrm{O}-\mathrm{H}$ stretch, $\mathrm{C}-\mathrm{H}$ stretch, and $\mathrm{CH}_{2} / \mathrm{CH}_{3}$ bend decreases. This reproduces the results in previous reports that PVA molecule undergoes elimination reaction and hydroxyl groups decrease $[9,12]$. Subsequently, some $\mathrm{C}-\mathrm{OH}$ groups theoretically form double bonds $\mathrm{C}=\mathrm{O}$ causing the reduction of $\mathrm{CH}_{2}$ and $\mathrm{C}-\mathrm{H}$ stretch, while some may lead to polymer dehydration with the formation of ether groups between PVA molecules, which is shown by the changes in peak around $\mathrm{C}-\mathrm{O}$ stretch. Here, $\mathrm{C}=\mathrm{O}$ stretching of the $\mathrm{P} 8$ sample shows the existence of acetate groups. PVA is the product of the hydrolysis process of polyvinyl acetate. This means that the reaction may be incomplete leaving residual acetate groups [12]. Therefore, the changes at this peak may be the replacement of acetate group with ketone or aldehyde group. Notably, the absorbance of $\mathrm{C}-\mathrm{O}$ stretch does not follow the order of exposure time. This phenomenon expresses the relationship between the reduction of $\mathrm{C}-\mathrm{OH}$ groups and the formation of C-O groups with respect to time of exposure. To be specific, when PVA is treated with microwave, an increase of $\mathrm{C}=\mathrm{O}$ groups parallelly reduces $\mathrm{C}-\mathrm{OH}$ group, thus lowering the absorbance; however, after $90 \mathrm{~s}$ of microwave exposure, ether group C-O form and its absorbance of light is higher and enhances the absorbance at peak $1088 \mathrm{~cm}^{-1}$.

Figure 1 depicts the FTIR spectra of freeze-dried PVA/silver nitrate solution with the concentration of $6 \mathrm{wt} \%$, $8 \mathrm{wt} \%$, and $10 \mathrm{wt} \%$ under different periods of microwave irradiation. The intensities of $\mathrm{O}-\mathrm{H}$ bonds as well as other bonds correlate with the concentration of PVA in the microwaved $\mathrm{PVA} /$ silver nitrate solutions, except for C-O peaks after $90 \mathrm{~s}$ of microwave exposure. It can be considered that the crosslinking reaction of PVA molecules is limited even when the concentration of PVA increases. As mentioned above, after $90 \mathrm{~s}$ of microwave exposure, the ether bridges between PVA molecules form. However, compared between $60 \mathrm{~s}$ and $90 \mathrm{~s}$

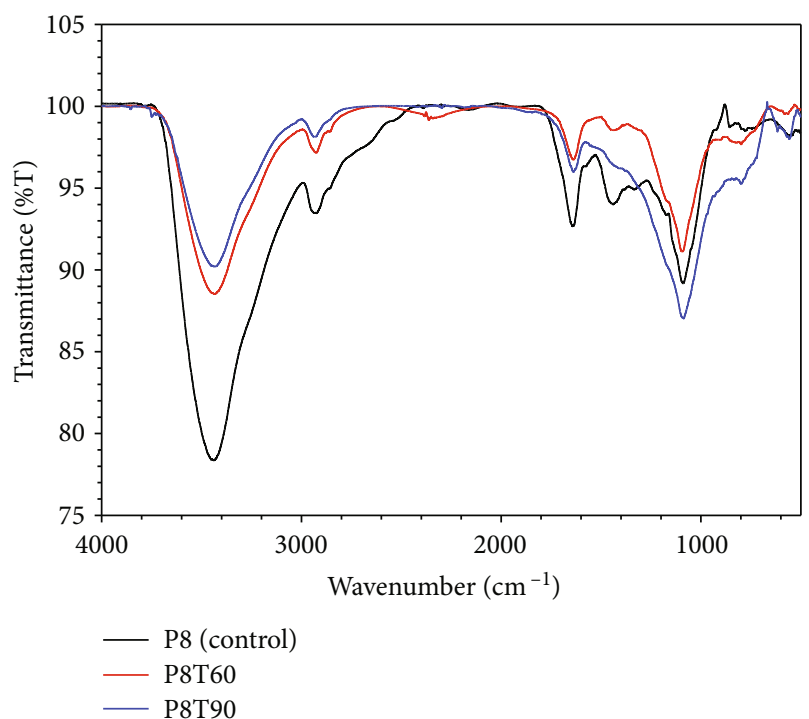

FIgURe 3: FTIR spectra of P8, P8T60, and P8T90.

of microwave exposure, the PVA/ $\mathrm{Ag}^{+}$solution is provided with more energy after being treated with microwave for $90 \mathrm{~s}$; however, C-O peak of P8T90Ag matches that of P10T90Ag. The reason could be that the spatial structure of conjugated PVA prevents themselves from further intermolecular reaction. In other words, the $\mathrm{O}-\mathrm{H}$ group is continuously broken, but the rate of $\mathrm{C}-\mathrm{O}$ ether group formation is decreased, causing the equal transmittance at peak $1088 \mathrm{~cm}^{-1}$ (as shown in Figure 2).

Figure 2 illustrates the FTIR results of PVA at $8 \mathrm{wt} \%$ under microwave irradiation at $60 \mathrm{~s}$ and $90 \mathrm{~s}$ with or without adding the $\mathrm{AgNO}_{3}$ solution. After $60 \mathrm{~s}$ or $90 \mathrm{~s}$ of microwave exposure, the transmittances of $\mathrm{O}-\mathrm{H}$ stretching of samples having $\mathrm{Ag}^{+}$(P8T60Ag and $\left.\mathrm{P} 8 \mathrm{~T} 90 \mathrm{Ag}\right)$ are lower than those 
of samples not having $\mathrm{Ag}$ (P8T60 and P8T90). This means that at the same time of microwave exposure, $\mathrm{Ag}^{+}$in PVA solution prevents the degradation of PVA. It may be caused by the redox reaction of $\mathrm{Ag}^{+}$and PVA when part of the energy provided by microwave process may be used for the redox reaction. Moreover, the existence of Ag does not affect the formation of ether bridges as shown in the C-O stretching of those samples.

Stability of nanoparticles is strongly dependent on the viscosity of the gels. When PVA is considered itself, the crosslinking between PVA molecules by ether groups is the only chemical parameter; however, it plays a minor role due to its limitation of spatial complexity. This promotes the combination of PVA with other polymers to crosslink instead of PVA itself.

\section{Conclusion}

The aim of this work is to investigate the chemical changes in $\mathrm{PVA} / \mathrm{Ag}^{+}$solution under a short period of microwave irradiation. The results have shown that PVA under microwave is a potential method to synthesize AgNPs. Within a short period of time, PVA still maintains O-H groups, which is the basis of its properties. Ether bridges, intermolecular bonds, are considered minor impacts on PVA crosslinking because of their limitation in spatial structure. The existence of $\mathrm{Ag}^{+}$reduces the rate of hydrolysis of PVA.

\section{Data Availability}

The data used to support the findings of this study are included within the article.

\section{Conflicts of Interest}

The authors declare that there is no conflict of interest regarding the publication of this paper.

\section{Acknowledgments}

The facilities for this research are supported by International University-Vietnam National University.

\section{References}

[1] K. Zheng, M. I. Setyawati, D. T. Leong, and J. Xie, "Antimicrobial silver nanomaterials," Coordination Chemistry Reviews, vol. 357, pp. 1-17, 2018.

[2] H. Bahadar, F. Maqbool, K. Niaz, and M. Abdollahi, "Toxicity of nanoparticles and an overview of current experimental models," Iranian Biomedical Journal, vol. 20, no. 1, pp. 1-11, 2016.

[3] M. Yasuyuki, K. Kunihiro, S. Kurissery, N. Kanavillil, Y. Sato, and Y. Kikuchi, "Antibacterial properties of nine pure metals: a laboratory study using Staphylococcus aureus and Escherichia coli," Biofouling, vol. 26, no. 7, pp. 851-858, 2010.

[4] R. Vazquez-Muñoz, B. Borrego, K. Juárez-Moreno et al., "Toxicity of silver nanoparticles in biological systems: does the complexity of biological systems matter?," Toxicology Letters, vol. 276, pp. 11-20, 2017.
[5] W. H. De Jong, L. T. M. Van Der Ven, A. Sleijffers et al., "Systemic and immunotoxicity of silver nanoparticles in an intravenous 28 days repeated dose toxicity study in rats," vol. 34, 2013.

[6] M. Akter, M. T. Sikder, M. M. Rahman et al., "A systematic review on silver nanoparticles-induced cytotoxicity: physicochemical properties and perspectives," Journal of Advanced Research, vol. 9, pp. 1-16, 2018.

[7] R. A. Franco, T. H. Nguyen, and B.-T. Lee, "Preparation and characterization of electrospun PCL/PLGA membranes and chitosan/gelatin hydrogels for skin bioengineering applications," Journal of Materials Science: Materials in Medicine, vol. 22, no. 10, pp. 2207-2218, 2011.

[8] T.-H. Nguyen, Y.-H. Kim, H.-Y. Song, and B.-T. Lee, "Nano Ag loaded PVA nano-fibrous mats for skin applications," Journal of Biomedical Materials Research Part B: Applied Biomaterials, vol. 96B, no. 2, pp. 225-233, 2011.

[9] N. T. Hiep, H. C. Khon, V. V. T. Niem et al., "Microwaveassisted synthesis of chitosan/polyvinyl alcohol silver nanoparticles gel for wound dressing applications," International Journal of Polymer Science, vol. 2016, Article ID 1584046, 11 pages, 2016.

[10] T. T. Nhi, H. C. Khon, N. T. T. Hoai et al., "Fabrication of electrospun polycaprolactone coated with chitosan-silver nanoparticles membranes for wound dressing applications," Journal of Materials Science: Materials in Medicine, vol. 27, no. 10, pp. 156-156, 2016.

[11] T.-H. Nguyen, K.-H. Lee, and B.-T. Lee, "Fabrication of Ag nanoparticles dispersed in PVA nanowire mats by microwave irradiation and electro-spinning," Materials Science and Engineering: C, vol. 30, no. 7, pp. 944-950, 2010.

[12] A. Bernal, I. Kuritka, V. Kasparkova, and P. Saha, "The effect of microwave irradiation on poly(vinyl alcohol) dissolved in ethylene glycol," Journal of Applied Polymer Science, vol. 128, no. 1, pp. 175-180, 2013.

[13] J. P. Cook, G. W. Goodall, O. V. Khutoryanskaya, and V. V. Khutoryanskiy, "Microwave-assisted hydrogel synthesis: a new method for crosslinking polymers in aqueous solutions," Macromolecular Rapid Communications, vol. 33, no. 4, pp. 332-336, 2012. 


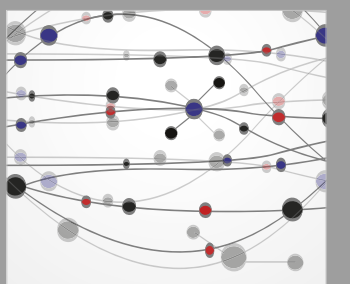

The Scientific World Journal
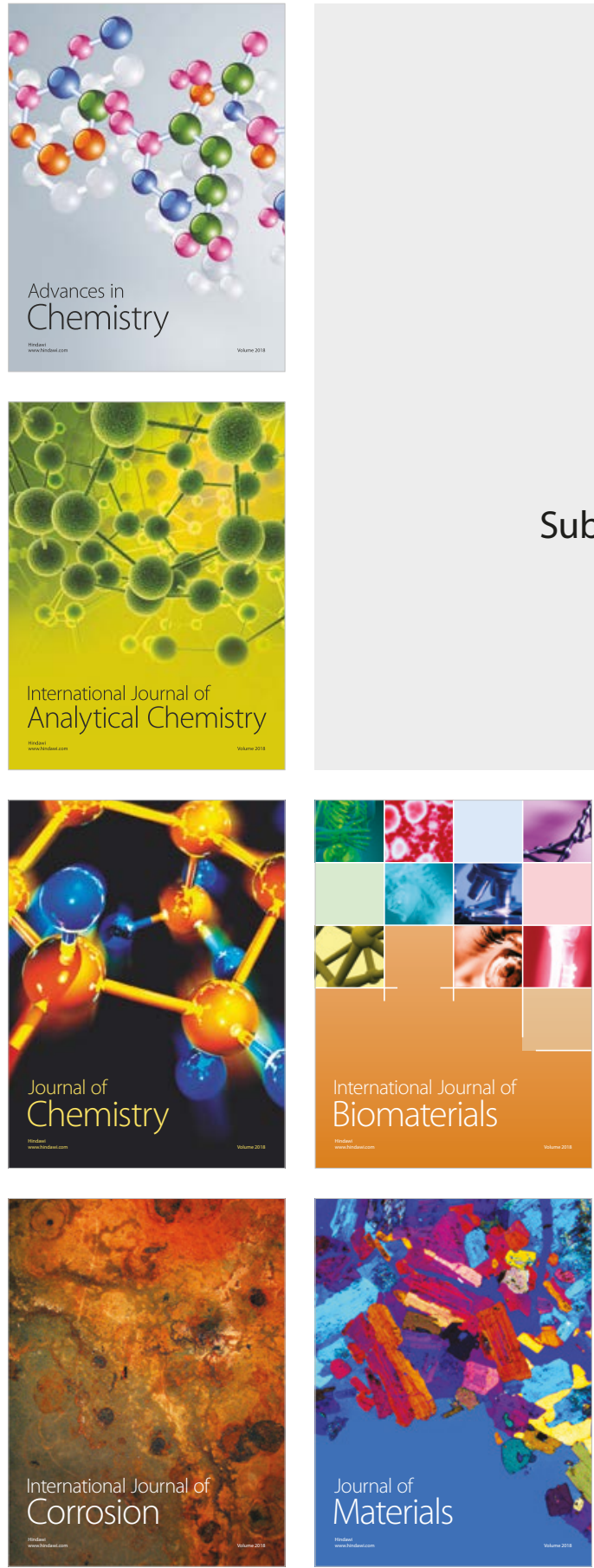

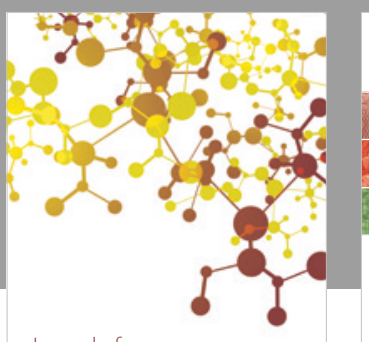

Journal of

Applied Chemistry
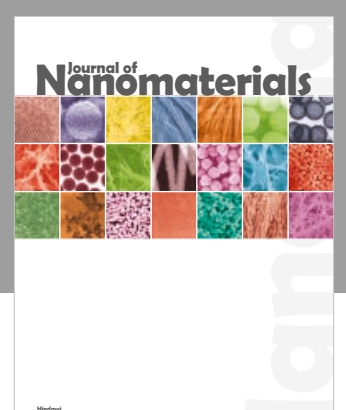

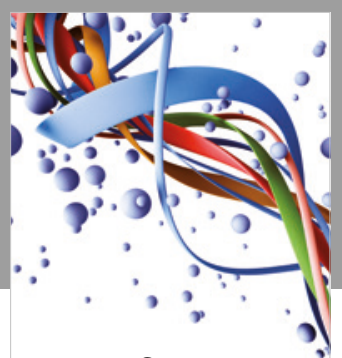

Scientifica

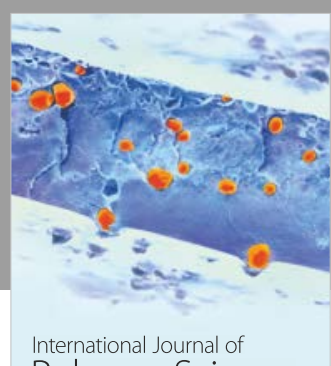

Polymer Science

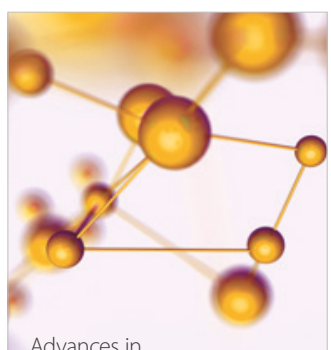

Physical Chemistry
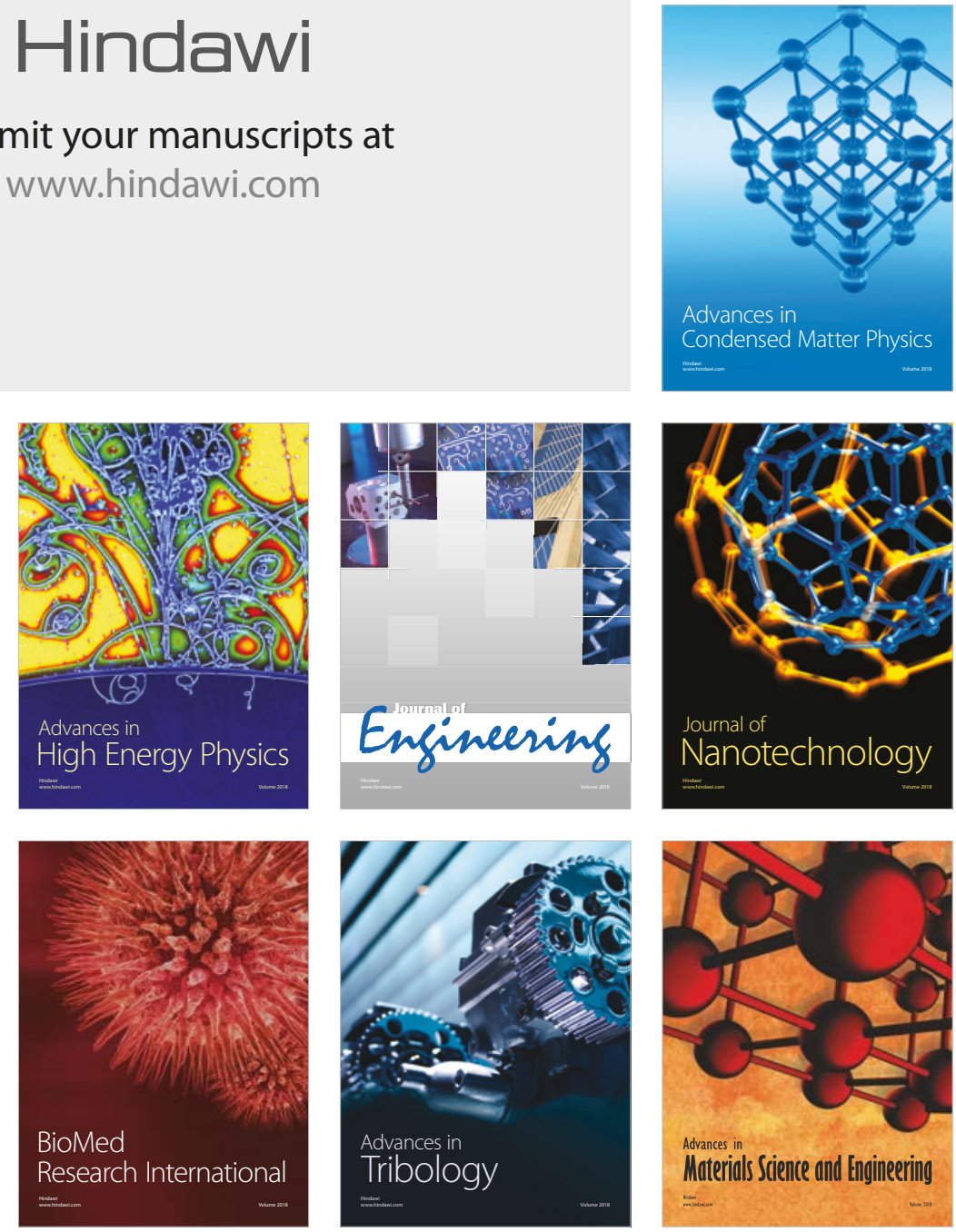